$15^{\text {th }}$ International Conference on

AEROSPACE SCIENCES \& AVIATION TECHNOLOGY, $\boldsymbol{A S A T}$ - 15 - May 28 - 30, 2013, Email: asat@mtc.edu.eg, Military Technical College, Kobry Elkobbah, Cairo, Egypt, Tel: +(202) 24025292 -24036138, Fax: +(202) 22621908

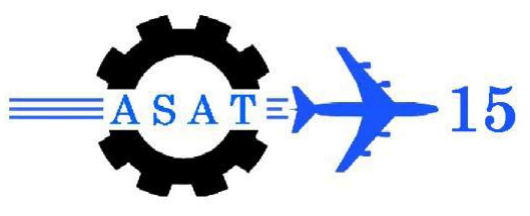

\title{
Flow-Sound Interaction Mechanism of Two Side-by-Side Cylinders in Cross-Flow
}

\author{
A. Mohany ${ }^{*}$ and M. Hassan ${ }^{\dagger}$
}

\begin{abstract}
A numerical simulation of the flow-excited acoustic resonance for the case of two side-by-side cylinders in cross-flow is performed. One spacing ratio between the cylinders, $\mathrm{T} / \mathrm{D}=2.5$, is investigated, where $\mathrm{D}$ is the diameter of the cylinders and $\mathrm{T}$ is the center-tocenter distance between them. The unsteady flow field is simulated using a finite-volume method at a Reynolds number of 30000 . This simulation is then coupled with a finite element simulation of the resonant sound field, by means of Howe's theory of aerodynamics sound, to reveal the details of flow-sound interaction mechanisms, including the nature and the locations of the aeroacoustic sources in the flow field. At the off resonance conditions the vortex shedding from the cylinders occurs at a single frequency with a Strouhal number of 0.217. Moreover, a symmetric wake structure downstream of the two cylinders is observed. However, when acoustic resonance is initiated the symmetric wake structure downstream of the cylinders is eliminated and the vortex shedding from both cylinders becomes antisymmetric. Moreover, two main aeroacoustic sources located just downstream of each cylinder are observed. The numerical results are compared with the experimental results presented in a previous investigation and favourable agreement is obtained.
\end{abstract}

Keywords: Acoustic Resonance, two side-by-side cylinders, wake structure, vortex shedding, aeroacoustic sources

\section{Nomenclature}

c speed of sound

$\mathrm{C}_{\mathrm{L}} \quad$ lift coefficient

$\mathrm{C}_{\mathrm{D}} \quad$ drag coefficient

$\mathrm{C}_{\mathrm{Lrms}}$ root mean square amplitude of oscillating lift coefficient

D cylinder diameter

$\mathrm{f}_{\mathrm{a}} \quad$ frequency of the lowest acoustic resonance mode of the test section $(=\mathrm{c} / 2 \mathrm{~W})$

$\mathrm{f}_{\mathrm{v}} \quad$ vortex shedding frequency

$\mathrm{H}$ height of the duct

M Mach number

$\mathrm{P} \quad$ acoustic pressure

$\mathrm{P}_{\mathrm{rms}} \quad$ root mean square amplitude of acoustic pressure.

$\mathrm{P}^{*} \quad$ normalized sound pressure

St Strouhal number of vortex shedding

$\mathrm{T}$ center-to-center distance between the cylinders

Assistant Professor, University of Ontario Institute of Technology, Ontario, Canada; atef.mohany@uoit.ca

$\dagger \quad$ Associate Professor, University of Guelph, Ontario, Canada; mahassan@ uoguelph.ca 


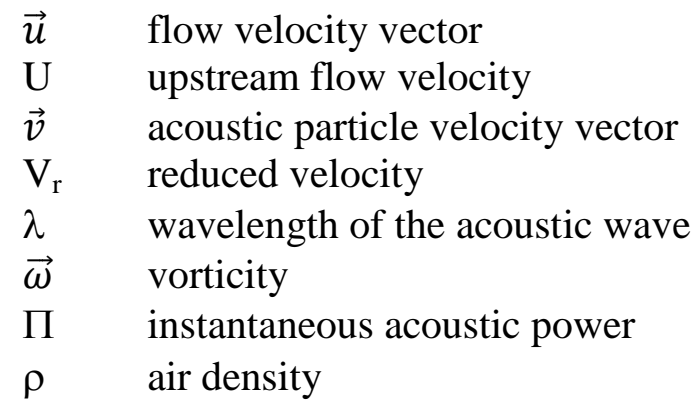

\section{Introduction}

The phenomenon of flow-excited acoustic resonance phenomenon, where the flow surrounding a bluff body vibrates in a resonant manner, is a design concern in many engineering applications, especially when wakes of bluff bodies are encountered in ducts, piping systems or other confined volumes. It causes noise and vibration problems and could lead to fatigue failure to the equipment. This is because when these resonances are excited, the resulting acoustic pressure may exceed the dynamic head of the mean flow. A crucial event in the mechanism of acoustic resonance is the ability of sound to modulate, and essentially "lock-in", the process of vortex shedding. While this phenomenon is relatively well understood for the case of isolated cylinders, Blevins (1985), Mohany and Ziada (2005) and Mohany (2013), there are many unresolved issues for the more complex case of multiple cylinders in close proximity.

Mohany and Ziada (2005, 2009a, 2009b, 2009c) investigated experimentally and numerically the excitation mechanism of acoustic resonance for the case of two tandem cylinders in crossflow. They found that the aeroacoustic response of two-tandem cylinders in cross-flow can be considerably different from that of a single cylinder under similar flow conditions. For the case of two tandem cylinders, the acoustic resonance is excited over two different ranges of flow velocity; the pre-coincidence and the coincidence resonance ranges. For the coincidence resonance range, the main aeroacoustic source is found to be located just downstream of the downstream cylinder, and the excitation mechanism of this resonance range is found to be similar to that of a single cylinder. However, for the pre-coincidence resonance range, the primary acoustic source is found to be located in the gap between the cylinders.

Hanson et al. (2009) investigated experimentally the aeroacoustic response of two side-byside cylinders in cross-flow. They observed that for small and intermediate spacing ratios, $\mathrm{T} / \mathrm{D}<2.2$, the wakes exhibit bi-stable flow features as those reported in the literature, e.g. Bearman and Wadcock (1973), Kim and Durbin (1988) and Sumner et al. (1999). Two Strouhal numbers of vortex shedding are observed before the onset of acoustic resonance. However, during the experiments, self-excited acoustic resonance occurred at an intermediate Strouhal number which lies between those corresponding to the bi-stable flow regimes. Hanson et al. (2009) concluded that the resonant sound field synchronizes the flow in the wakes of the cylinders and thereby eliminates the bi-stable biased flow. For this reason, acoustic resonances occur at an intermediate Strouhal number, which approximates that corresponding to vortex shedding from side-by-side cylinders with large spacing. Mohany et al. (2011) conducted a numerical investigation of the flow-excited acoustic resonance for two side-by-side cylinders in cross-flow with a spacing ratio of $T / D=1.25$. Before the excitation of acoustic resonance, Mohany et al. (2011) observed a bi-stable flow regime in the wake structure behind the two cylinders. Moreover, the Strouhal numbers corresponding to the structures in the narrow and wide wakes are 0.24 and 0.11 , respectively. However, after the excitation of acoustic resonance, the flow-field is coupled with the acoustic field at a Strouhal 
number between those observed before the onset of acoustic resonance. The numerical results of Mohany et al. (2011) agree favourably with the experimental results of Hanson et al. (2009).

The main objective of this paper is to extend the work of Mohany et al. (2011) to cover two side-by-side cylinders with a larger spacing ratio, $\mathrm{T} / \mathrm{D}=2.5$ to reveal the details of flowsound interaction mechanism, including the nature and the locations of the aeroacoustic sources. Better understanding of the flow-sound interaction mechanism and the resulting energy transfer between the flow and the resonant sound field would improve our ability to alleviate and control the occurrence of acoustic resonance. This can be achieved by identifying the location of the aeroacoustic sources in the flow field and developing suitable means to mitigate the energy transfer mechanism, Stoneman et al. (1988).

\section{Numerical Technique}

First, the flow field and the sound field are simulated separately (i.e. uncoupled). In order to simulate the coupling phenomenon, the simulation of the unsteady flow field was repeated with applying an oscillatory cross-flow velocity perturbation, which simulates the acoustic particle velocity of the resonant sound field.

The aeroacoustic sources (or sinks) in the flow field are then predicted by combining the solution of the acoustic field, which is modeled by the finite-element method using ABAQUS, with the acoustically coupled unsteady flow field, which is modeled using the Spalart-Allmaras (S-A) turbulent model in FLUENT to predict the vorticity distribution in the cylinder wake. Howe's theory of aerodynamic sound (1980, 1998), Equation 1, is then used to calculate the instantaneous acoustic power, $n$, generated by the convection of the unsteady vorticity field, a, within the sound field. In Equation 1, v stands for the acoustic particle velocity vector of the sound filed, $\mathrm{u}$ is the local velocity vector and the integral is performed over the fluid volume $\mathrm{V}$ containing the unsteady vorticity field. The net acoustic energy is the integration of the instantaneous acoustic power over a complete acoustic cycle. Therefore, the locations within the flow field where the acoustic energy is either absorbed or generated can be identified.

$$
\Pi=-\rho \int \vec{\omega} \cdot(\vec{u} \times \vec{v}) d \forall
$$

The key factor to understand how the energy could be transferred from the flow to the sound field, i.e. aerodynamic sound sources, or from the sound field to the flow, i.e. sinks, is the triple product $\vec{\omega} \cdot(\vec{u} \times \vec{v})$. The physical interpretation of the triple product could be explained with the aid of figure 1 . Consider a simple case in which the flow velocity vector, $\vec{u}$, is perpendicular to the acoustic velocity vector, $\vec{v}$. The angle between these vectors is $90^{\circ}$ in one half of the acoustic cycle and is $270^{\circ}$ in the other half. Therefore, the cross product $(\vec{u} \times \vec{v})$ is a vector in the positive $\hat{k}$ direction for one half of the acoustic cycle and in the negative $\hat{k}$ direction for the other half. Since the vorticity vector is a vector in $\mathrm{k}$ direction, the triple product for this simple case will be either a positive or a negative value. If the vorticity vector is constant over a complete acoustic cycle, the value of this triple product is oscillatory over the time because of the oscillation of the acoustic particle velocity. Hence, if the amplitudes of these vectors are constant, the total energy transfer over a complete cycle would be zero. Only in the case that the integral of the triple product is non-zero, the vorticity field could represent an acoustic source or sink. 

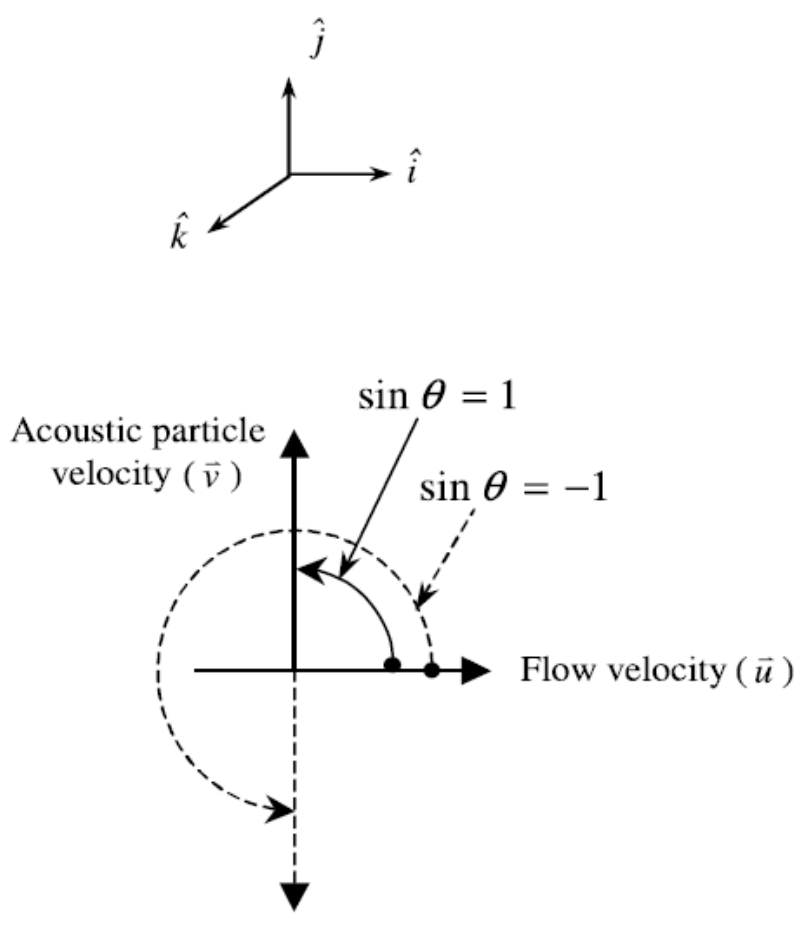

Figure 1: Physical interpretation of the triple product.

\section{Flow Field}

The simulation of the flow field was performed in FLUENT using the S-A turbulent model. This is a one equation turbulent model, which was developed specifically for aerospace applications involving wall bounded flows (Spalart and Allmaras, 1994). This model has proven to be advantageous in solving boundary layers with adverse pressure gradients (Isaev et al. 2005). Moreover, it is sufficiently robust for relatively crude simulations on coarse meshes, which allows the computation time to be reduced without sacrificing the accuracy of results. Therefore, the S-A model is believed to capture the flow field around circular cylinders with reasonable accuracy.

The simulation of the flow was performed at a Reynolds number of 30000 and assuming a two-dimensional flow. Although it might appear that the two-dimensional assumption is not valid, especially at a such high Reynolds number, previous experiments have shown that during acoustic resonance the flow oscillation is predominantly two-dimensional because the resonant sound filed synchronizes the vortex shedding along the cylinder span, Blevins (1985). Thus, simulating two-dimensional flow during acoustic resonance excitation is a reasonable assumption to capture the underlying flow physics. The Reynolds number value of 30000 was chosen in order to perform the simulations at flow conditions comparable to those which prevailed in the experiments reported by Hanson et al. (2009). Based on this Reynolds number, the mean flow velocity is $17.25 \mathrm{~m} / \mathrm{s}$, for a cylinder diameter of $25.4 \mathrm{~mm}$. The working medium is air with a density of $\rho=1.225 \mathrm{~kg} / \mathrm{m}^{3}$, and a viscosity of $\mu=1.7894 \times 10^{-5}$ $\mathrm{kg} / \mathrm{m}$.s. The simulation boundaries are placed at $\pm 10 \mathrm{D}$ in the cross-flow direction, 5D upstream and 20D downstream of the cylinder. Unstructured triangular mesh was used with a 123066 triangular cells. The triangular mesh was selected in order to enhance the interpolation accuracy when mapping the data from the acoustic field with triangular elements to the flow domain. The smallest grid size is about $1.064 \times 10^{-4} \mathrm{~m}^{2}$. Segregated (implicit) solver with a second-order discretization scheme was used throughout this simulation. For the velocity-pressure coupling, SIMPLEC algorithm was used, which generally improves the 
convergence for complicated flows involving turbulence. The time step size used for the computation is $2 \times 10^{-4} \mathrm{~s}$.

The lift force coefficients for this case are shown in figure 2. These coefficients are based on a flow simulation before applying the cross-flow oscillation. The FFT of the lift force coefficient of the top cylinder is shown in figure 3. A dominant frequency component at $147.5 \mathrm{~Hz}$ can be identified from this figure, which corresponds to a Strouhal number of about 0.217 . This agrees well with the value of 0.22 reported in the literature. The wake structure behind the two cylinders before applying the cross-flow oscillation is shown in figure 4, which shows clearly the bi-stable flow regimes.

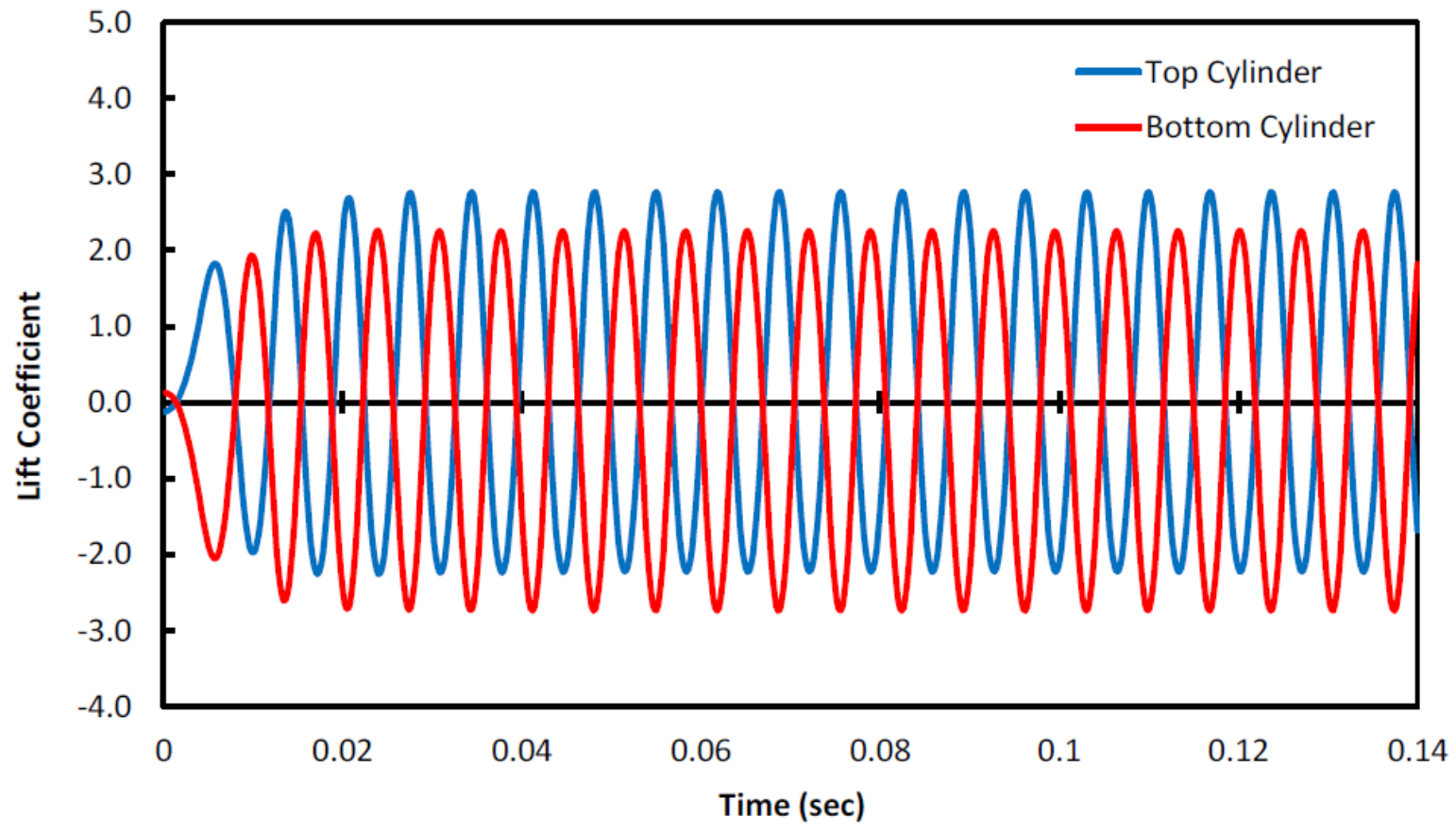

Figure 2: Lift coefficients for two side-by-side cylinders at $\mathbf{R e}=\mathbf{3 0 0 0 0}$, no acoustic excitation.

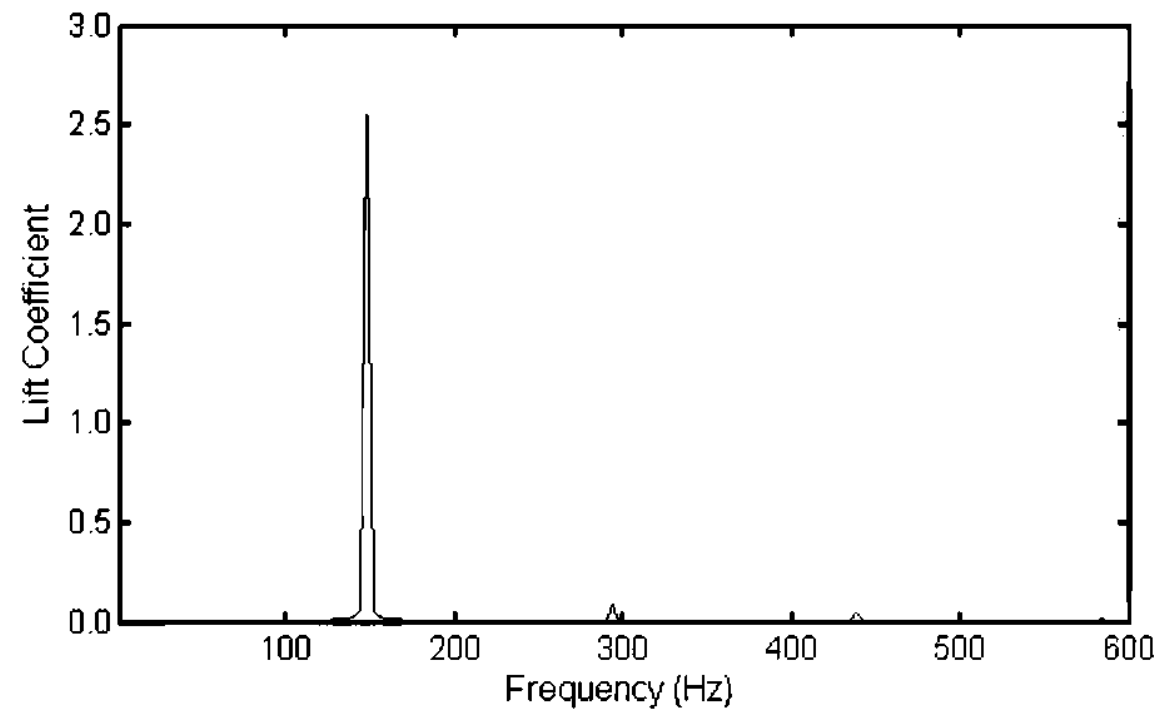

Figure 3: FFT of the lift coefficient on the top cylinder at $\operatorname{Re}=30000$, no acoustic excitation. 


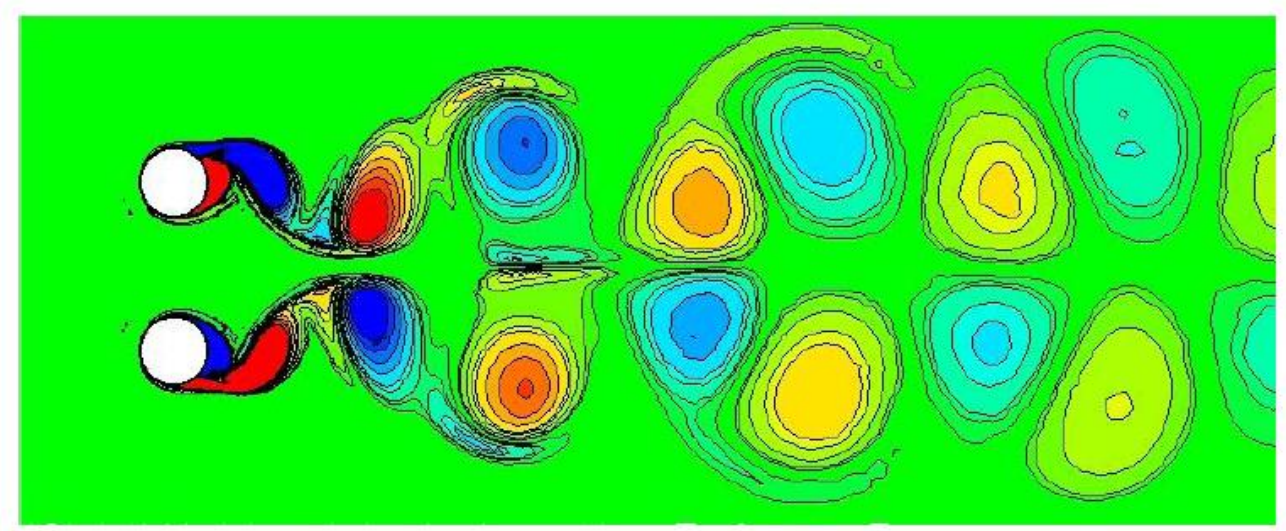

$t=0.4570 \mathrm{sec}$

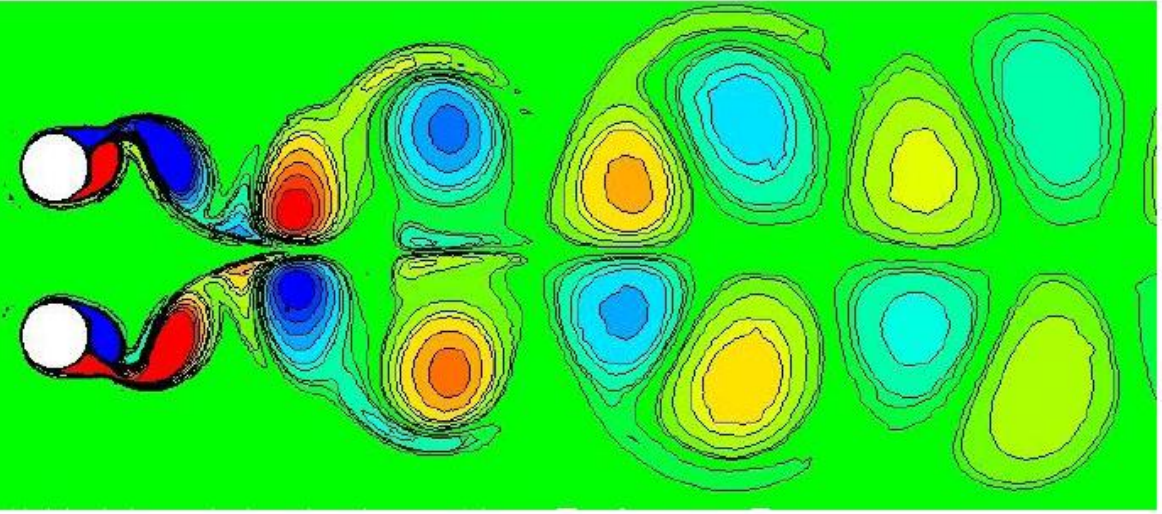

$\mathrm{t}=0.4578 \mathrm{sec}$

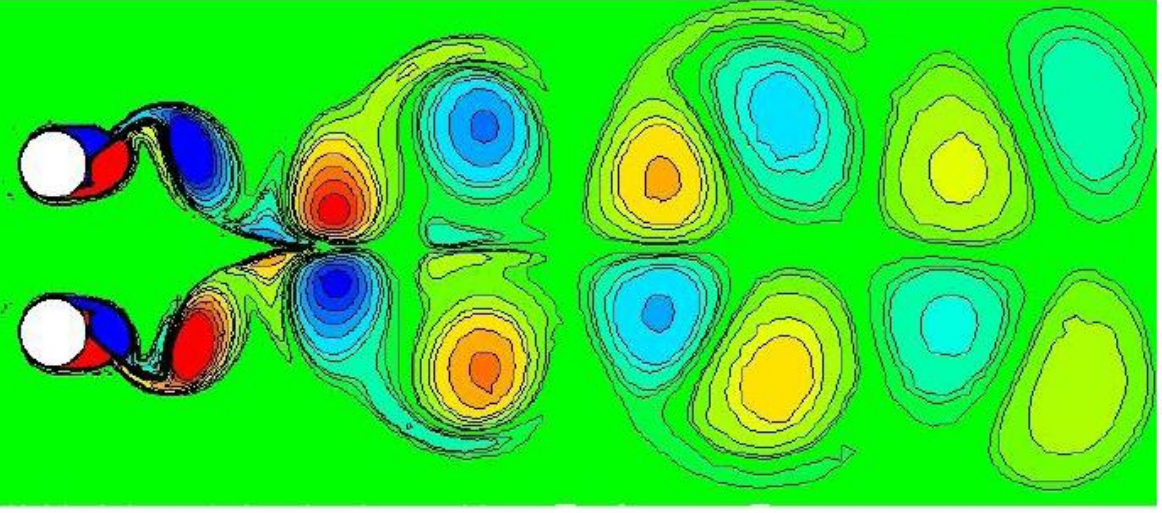

$t=0.4586 \mathrm{sec}$

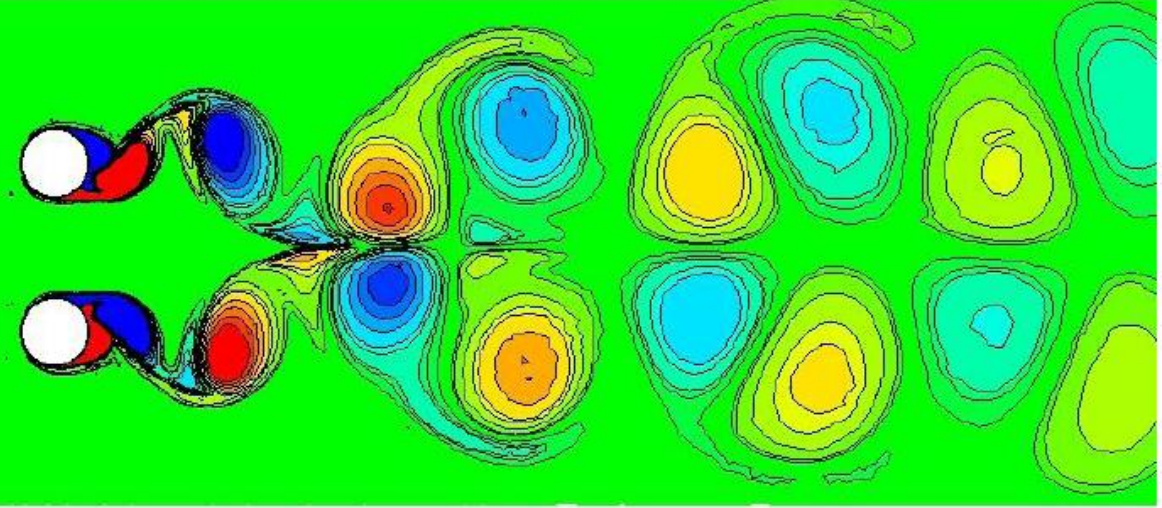

$t=0.4596 \mathrm{sec}$

Figure 4: Vorticity contours behind two side-by-side cylinders, $T / D=2.5$, at $\mathbf{R e}=30000$, no acoustic excitation. 


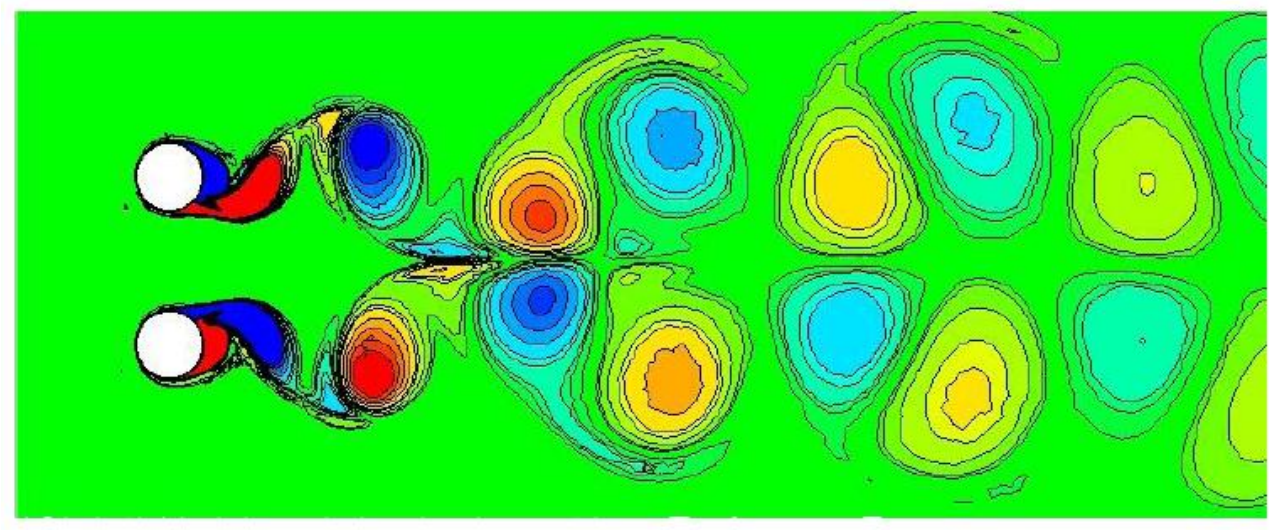

$t=0.4604 \mathrm{sec}$

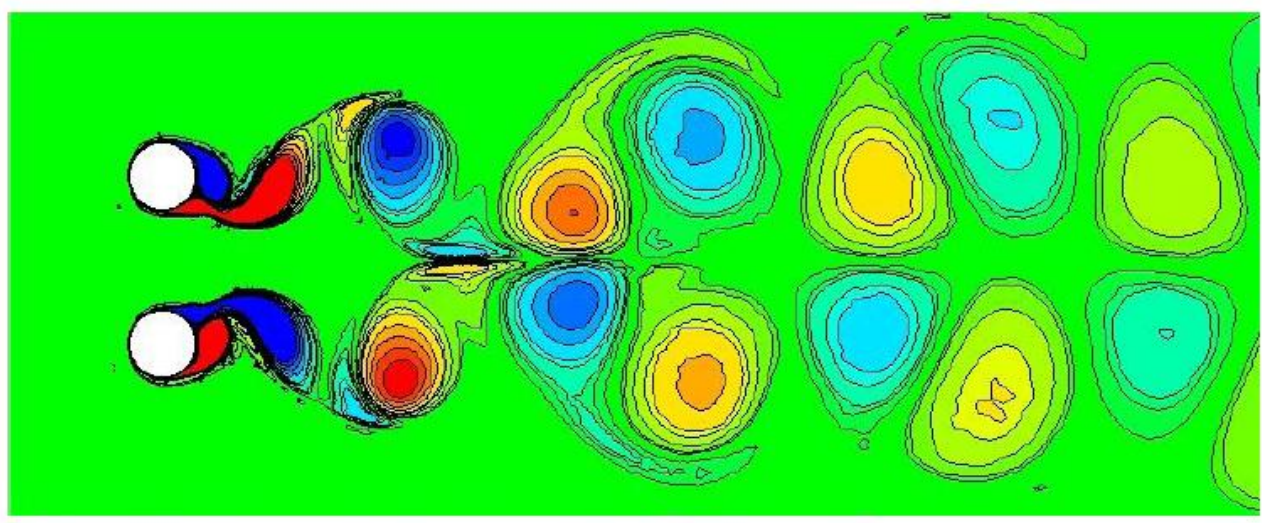

$t=0.4612 \mathrm{sec}$

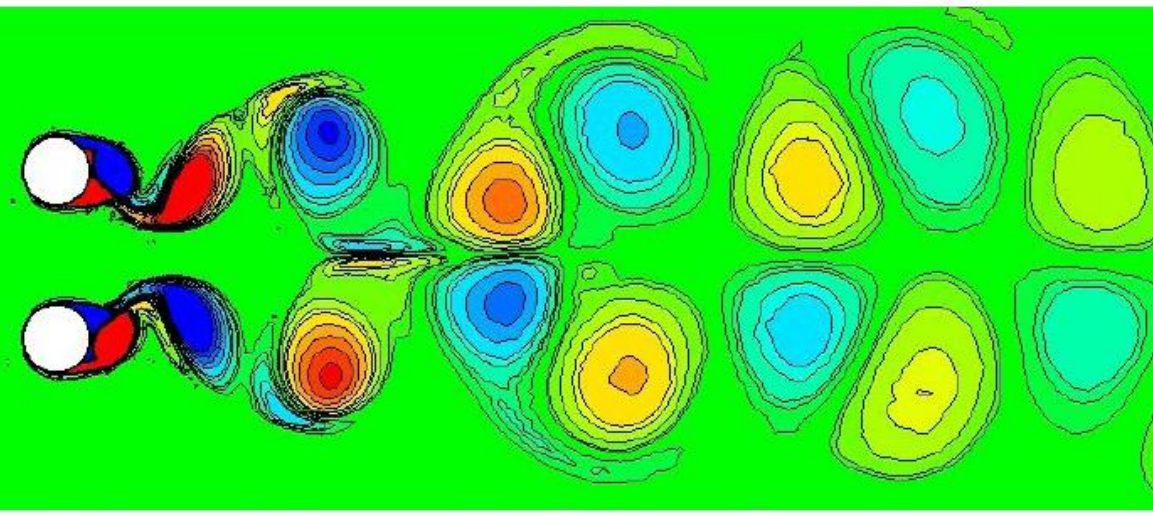

$t=0.4620 \mathrm{sec}$

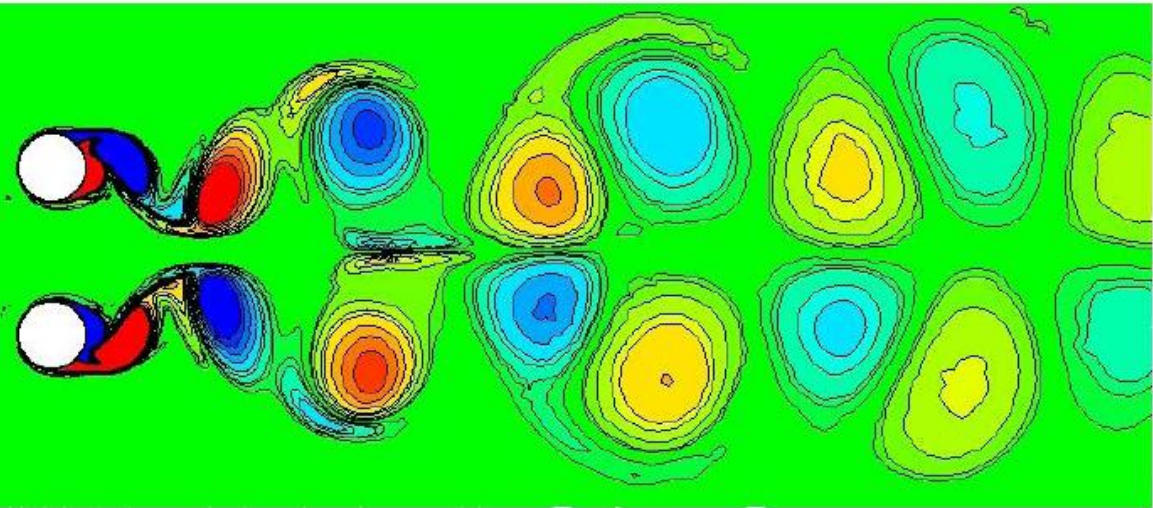

$t=0.4630 \mathrm{sec}$

Figure 4: (Continued) Vorticity contours behind two side-by-side cylinders, $T / D=2.5$, at $\operatorname{Re}=30000$, no acoustic excitation. 


\section{Acoustic Field}

A numerical simulation was performed to model the resonant sound field with the aid of the finite-element analysis in ABAQUS. The acoustic modes in the duct housing the cylinders oscillate with a simple harmonic motion in time. Therefore, the acoustic pressure of the resonant mode can be expressed as $p=\phi \mathrm{e}^{i(2 \pi f) t}$ and $\phi$ is a variable that satisfies the following Helmholtz equation (Kinsler et al. 2000),

$$
\nabla^{2} \phi+k^{2} \phi=0
$$

where $k=2 \pi f_{a} / c$ is the wavenumber and $c$ is the speed of sound. At the inlet and the outlet section of the duct, the boundary condition for the lowest acoustic mode is approximately zero acoustic pressure. The computation grid consisted of 3-node linear 2-D acoustic triangular elements. The acoustic particle velocity distribution was determined from the computed acoustic pressure field by means of Euler's equation:

$$
\rho \frac{\partial \vec{v}}{\partial t}=-\nabla p
$$

\section{Flow-Acoustic Coupling}

In order to perform the triple product and use Howe's theory of aerodynamic sound to determine the acoustic sources and sinks in the flow field, equation 1, it is crucial to determine first the phase relationship between the acoustic cycle and the vortex shedding cycle. This phase shift was obtained from the flow solver by monitoring the cross-flow oscillation at the boundaries and the lift force on the cylinders. This phase relationship is shown in figure 5. As it can be seen from this figure, the phase shift between the cross-flow oscillation and the flow field is about $84.7^{\circ}$. Therefore, the coupling between the flow field and the acoustic field was performed at this phase shift for a complete acoustic cycle. Moreover, it is clear from figure 5 that the lift coefficient on both the top and the bottom cylinders is in phase, which indicates that the wake structure downstream of the cylinders is anti-symmetric. The frequency of the acoustic cycle was taken to be $147.5 \mathrm{~Hz}$, which was equivalent to a Strouhal number of about 0.217 as discussed earlier.

It is important to mention that this coupling procedure is a one way coupling phenomenon, which means that the effect of the resonant sound field on the flow is fully accounted for, but the excitation of the sound field by the flow is not considered. This approach does not affect the nature or the locations of the sound sources or sinks for the given excitation amplitude. The main drawback of this one way coupling procedure is that the sound pressure level of the acoustic resonance can not be predicted. This is however not the objective of the present work.

The wake structure behind the cylinders after applying the cross-flow perturbation is shown in figure 6. A comparison between figures 4 and 6 , shows that after applying the cross-flow perturbation, the flow in the wakes of the cylinders becomes anti-symmetric. It is noteworthy to mention that the size of elements in the flow domain was much smaller than that used in 
the acoustic domain. However, the mesh for the acoustic simulation was constructed such that its nodal points were overlapped on the nodal points in the flow domain, as it is shown in figure 7. The triangular points in this figure are the nodal points in the acoustic domain and the circular points are the nodal points in the flow domain. Therefore, the triple product was performed over the nodal points of the acoustic mesh.

The instantaneous acoustic power was calculated every $45^{\circ}$ of the acoustic cycle (or vortex shedding cycle). The total acoustic energy is the integration of the instantaneous acoustic power over a complete acoustic cycle. Figure 8 shows the total acoustic energy over one cycle. The red colour in these figures represents net positive energy and the blue colour represents net negative energy. The total energy transfer per cycle for different downstream locations is shown in figure 9. For each streamwise location, the total energy represents the energy integrated over a rectangular area with a height extending along $\mathrm{y}= \pm 10 \mathrm{D}$ and a small width of $\Delta \mathrm{x}$ centered at the streamwise location $\mathrm{x}$. As it can be seen from this figure, the total energy transfer just downstream of the cylinder (the deep red area) is positive. Further downstream, there is almost periodic generation and absorption of acoustic energy.

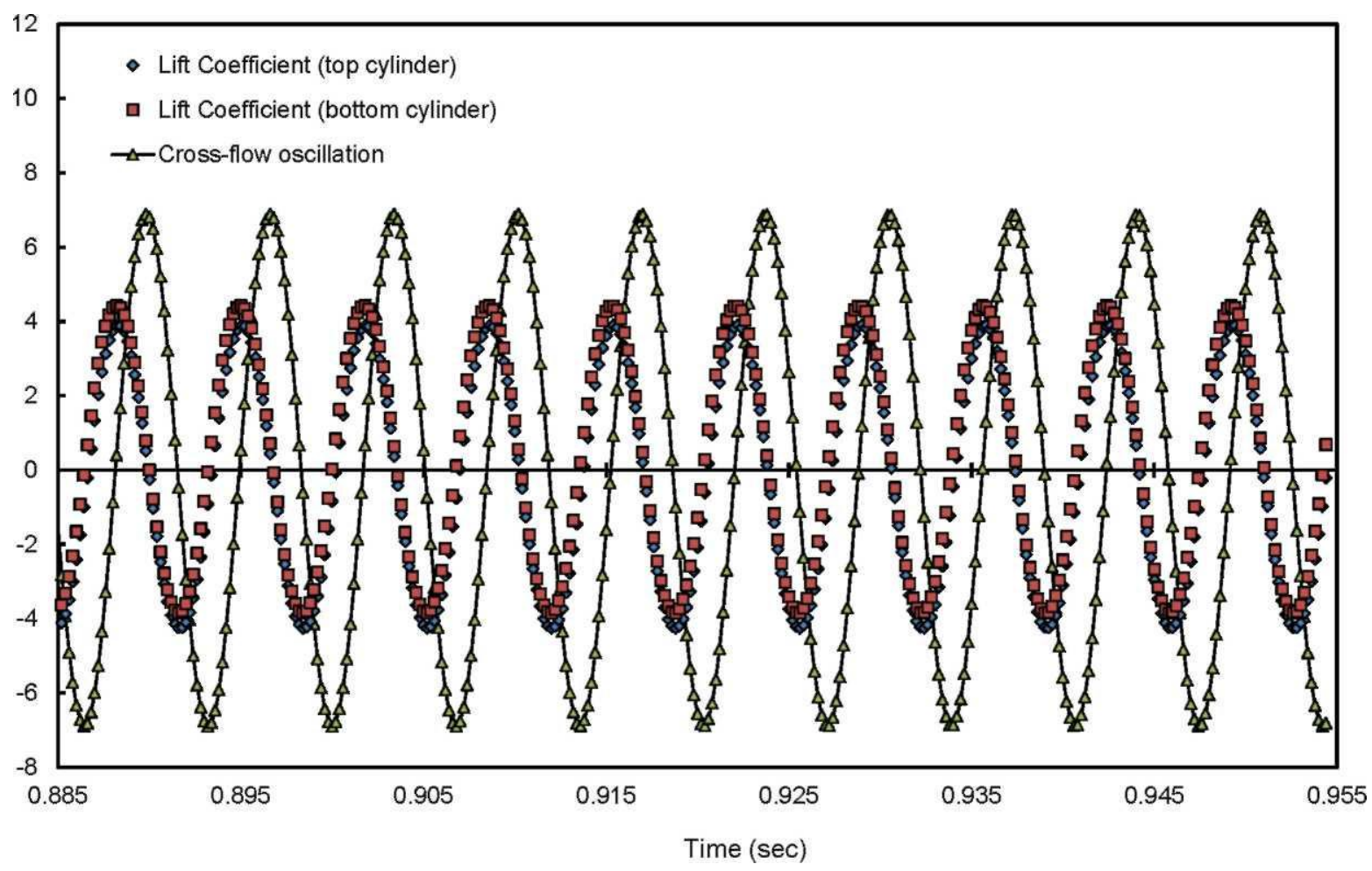

Figure 5: Phase relationship between the cross-flow oscillation and the lift coefficient on the top and bottom cylinders. 


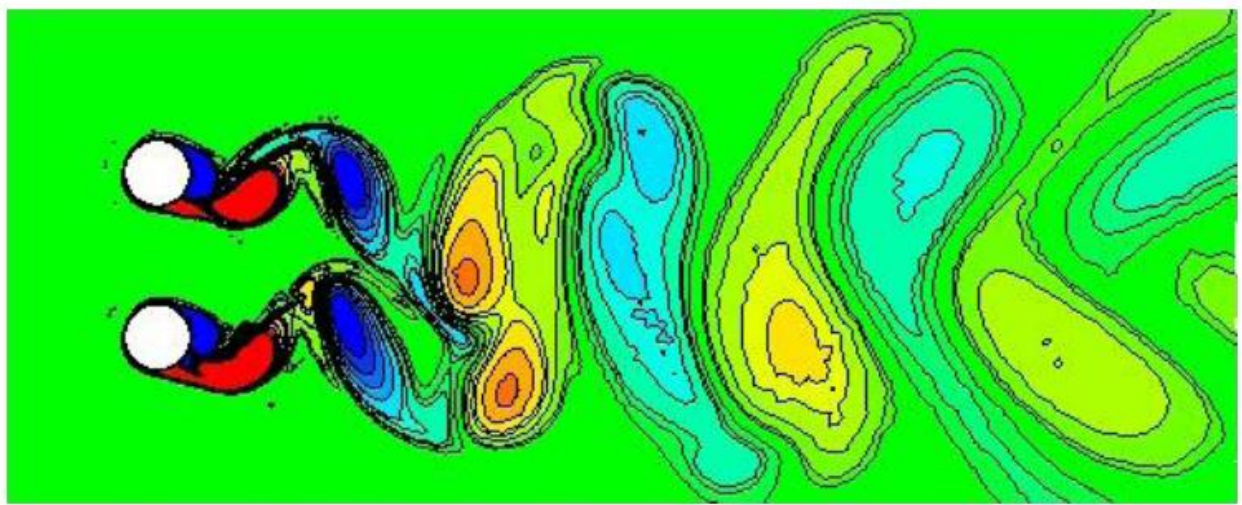

$\mathrm{t}=0.9560 \mathrm{sec}$

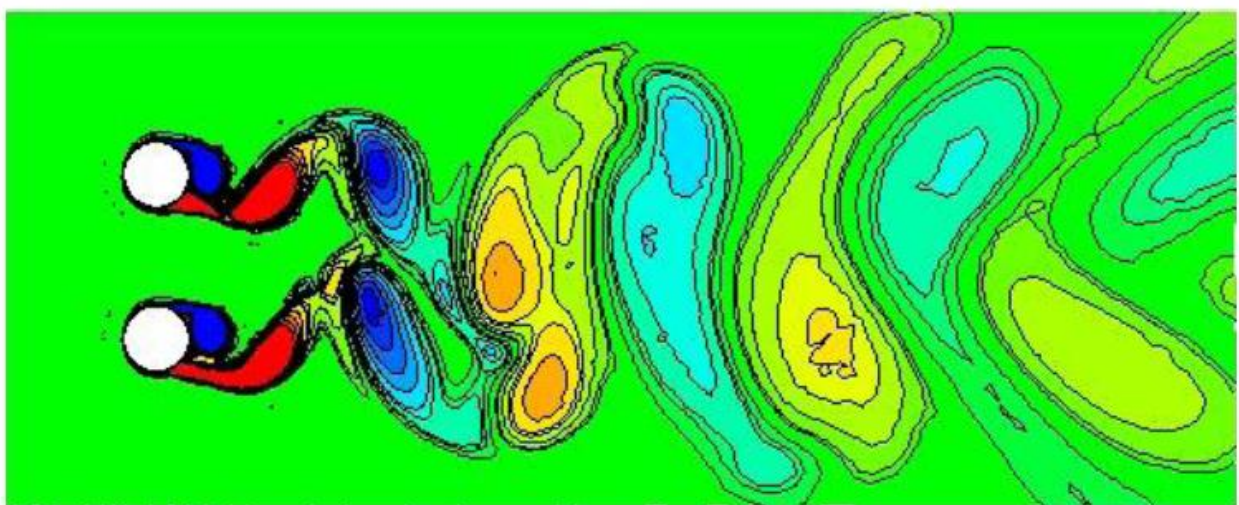

$t=0.9568 \mathrm{sec}$

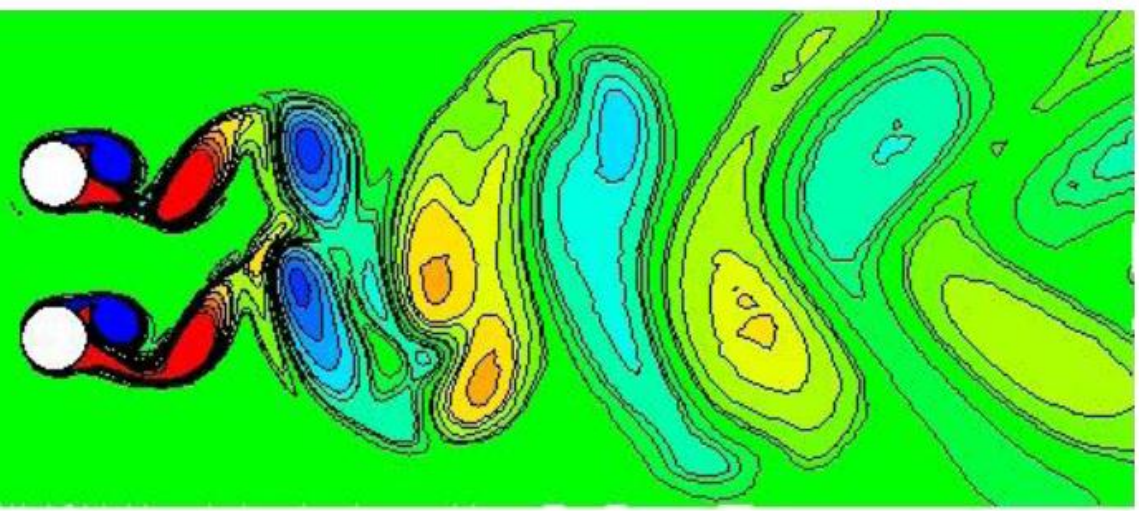

$\mathrm{t}=0.9576 \mathrm{sec}$

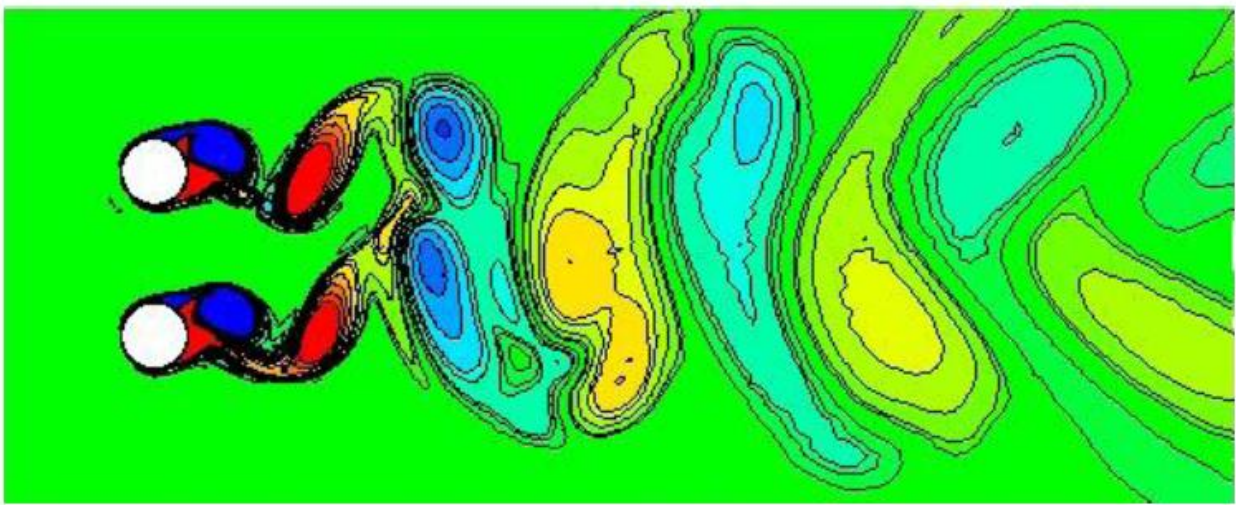

$t=0.9584 \mathrm{sec}$

Figure 6: Vorticity contours behind two side-by-side cylinders, $T / D=2.5$, at $\mathrm{Re}=\mathbf{3 0 0 0 0}$, after applying cross-flow perturbation, $v / \mathrm{U}=\mathbf{2 0 \%}$. 


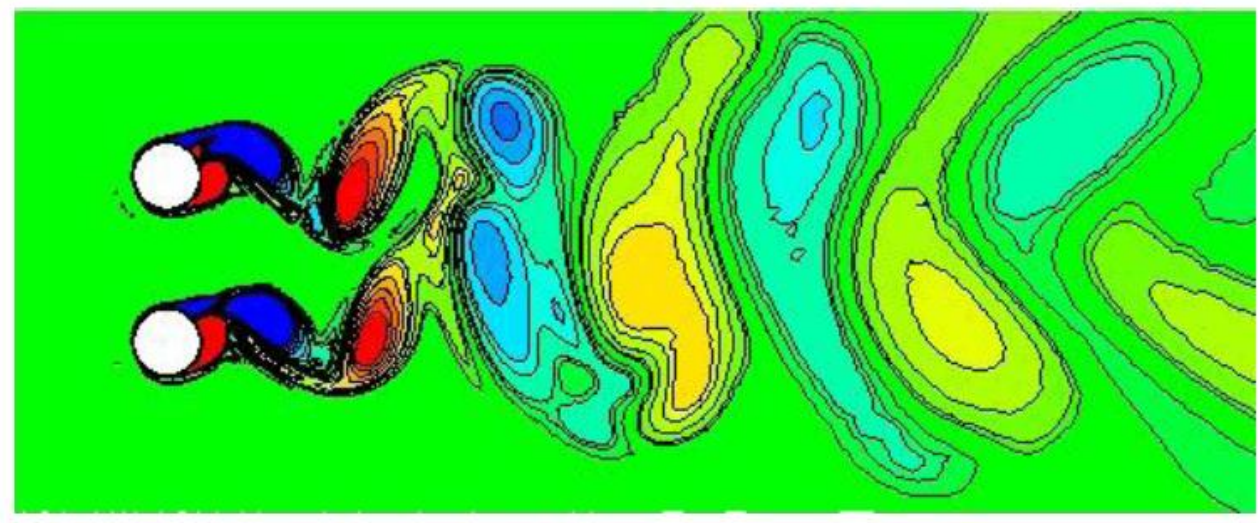

$t=0.9594 \mathrm{sec}$

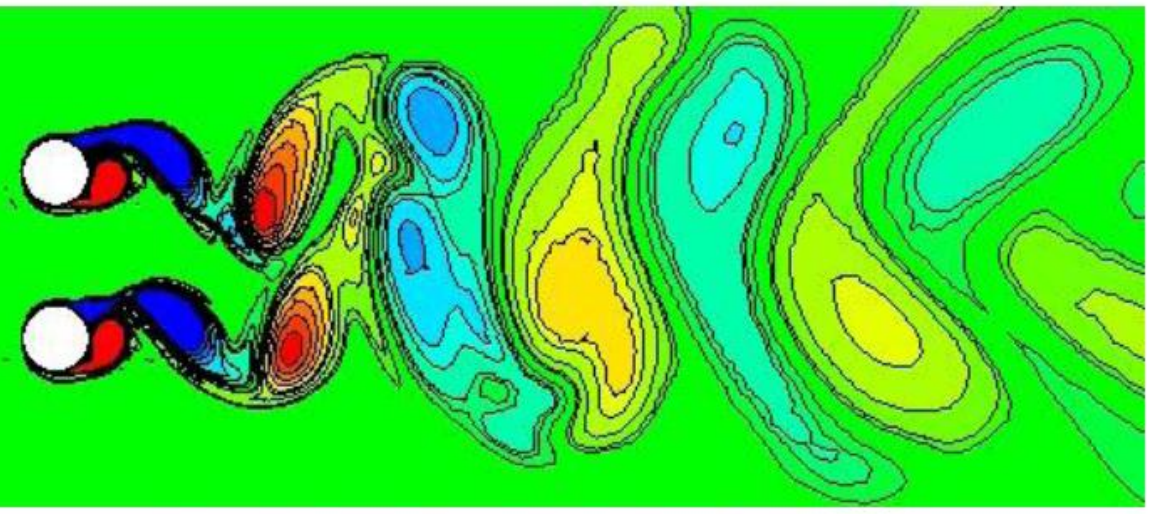

$t=0.9602 \mathrm{sec}$

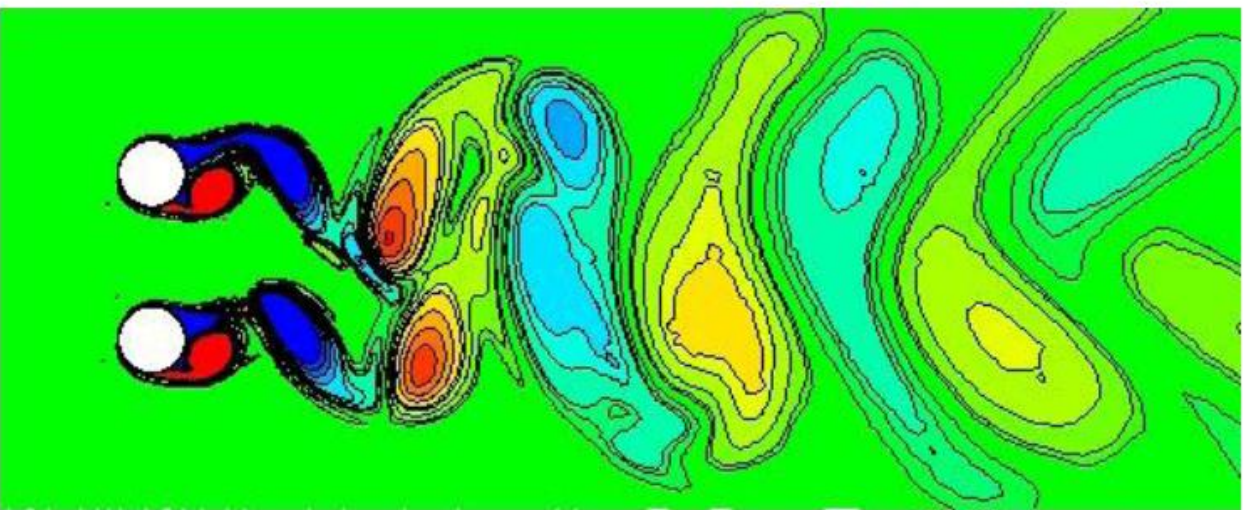

$t=0.9610 \mathrm{sec}$

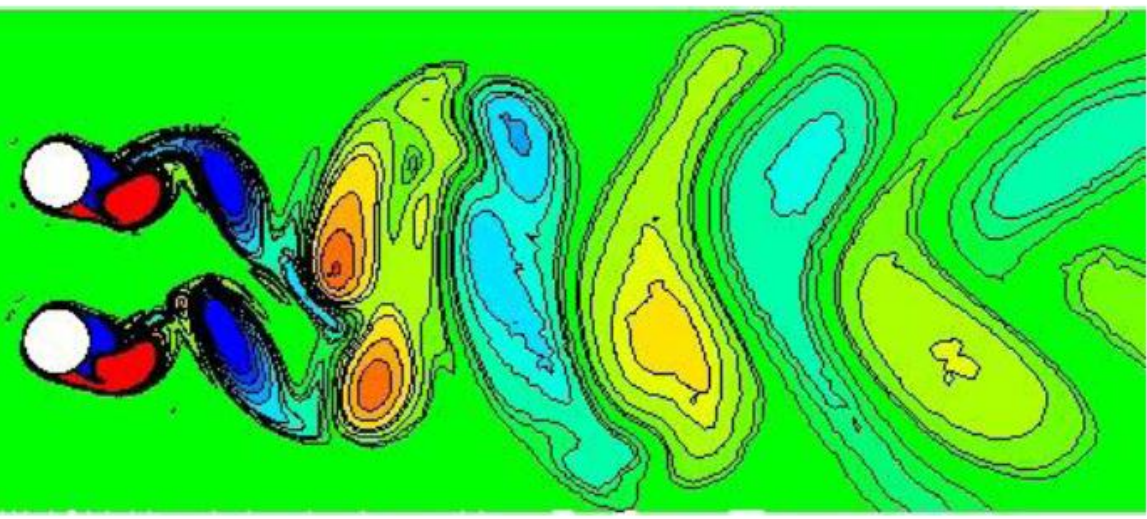

$t=0.9620 \mathrm{sec}$

Figure 6: (Continued) Vorticity contours behind two side-by-side cylinders, $T / D=2.5$, at $\mathrm{Re}=\mathbf{3 0 0 0 0}$, after applying cross-flow perturbation, $\mathrm{v} / \mathrm{U}=\mathbf{2 0 \%}$. 


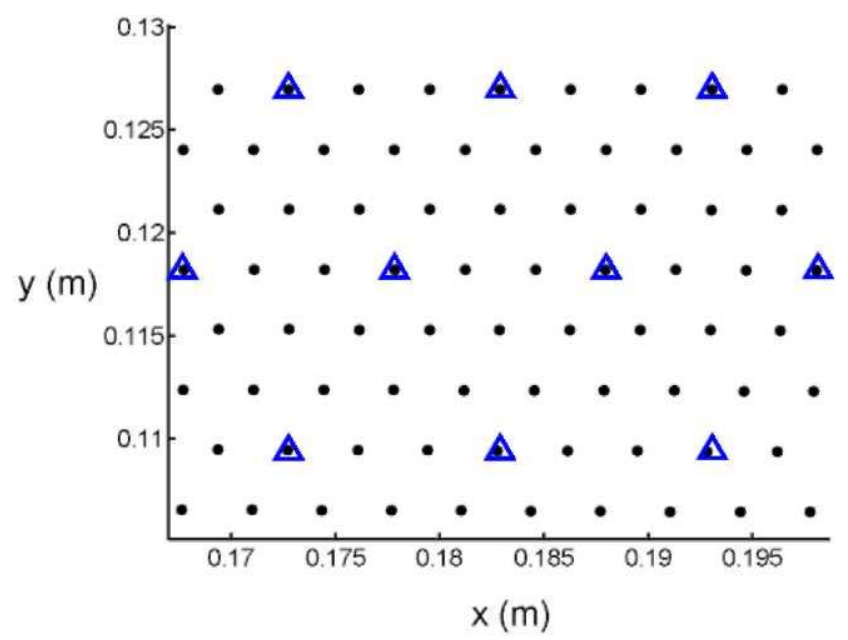

Figure 7: Small region in the simulation domain showing the nodal points of the acoustic and the flow meshes.

$\Delta$; acoustic nodal points, $\bullet$; flow nodal points.

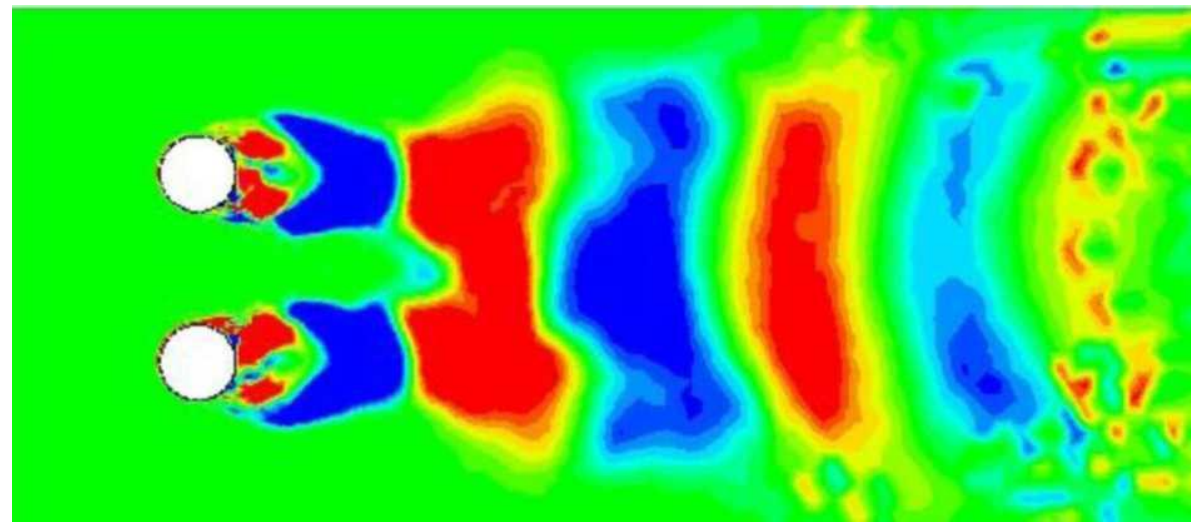

Figure 8: total acoustic energy over one cycle.

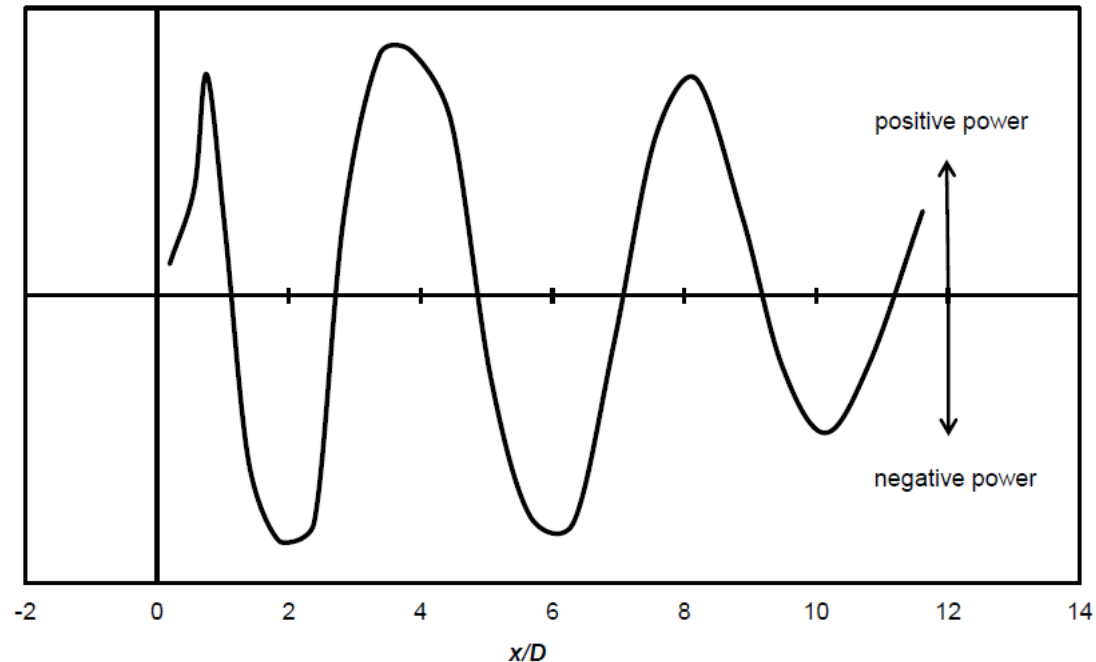

Figure 9: Total energy transfer per cycle at different downstream locations. 


\section{Conclusions}

A numerical simulation was performed to investigate the flow-excited acoustic resonance process for the case of two side-by-side cylinders in cross-flow. The spacing ratio between the cylinders is $\mathrm{T} / \mathrm{D}=2.5$. Reasonable agreement with the experimental results presented in Hanson et al. (2009) was obtained. Before applying the cross-flow oscillation, which mimics the excitation of acoustic resonance, a symmetric wake structure is observed behind the two cylinders. The Strouhal number at the off resonance condition is about 0.217 which agrees well with the experimental results presented in Hanson et al. (2009). After applying the crossflow perturbation, the flow in the wakes of the cylinders becomes anti-symmetric. Moreover, two strong aeroacoustic sources are observed in the wake of the two cylinders and they are located just downstream of each cylinder, resulting in a net positive energy transfer from the flow field to the sound field.

\section{References}

[1] Blevins, R. D., 1985, The effect of sound on vortex shedding from cylinders. Journal of Fluid Mechanics, 161: 217-237.

[2] Mohany, A. and Ziada, S., 2005, Flow-excited acoustic resonance of two tandem cylinders in cross flow. Journal of Fluids and Structures, 21: 103-119.

[3] Mohany, A., 2013, "Self-excited acoustic resonance of isolated cylinders in cross-flow" AECL Nuclear Review, Vol. 1, pp. 45 - 55.

[4] Mohany, A. and Ziada, S., 2009a, A parametric study of the resonance mechanism of two tandem cylinders in cross-flow. ASME Journal of Pressure Vessel Technology, $131,021302$.

[5] Mohany, A. and Ziada, S., 2009b, Effect of acoustic resonance on the dynamic lift forces acting on two tandem cylinders in cross-flow. Journal of Fluids and Structures, Volume 25, Issue 3, Pages 461-478.

[6] Mohany, A. and Ziada, S., 2009c, Numerical Simulation of the Flow-Sound Interaction Mechanisms of a Single and Two-Tandem Cylinders in Cross-Flow. ASME Journal of Pressure Vessel Technology, 131, 031306.

[7] Hanson, R., Mohany, A. and Ziada, S., 2009, Flow-excited acoustic resonance of two side-by-side cylinders in cross-flow. Journal of Fluids and Structures, Volume 25, Issue 1, Pages 80-94.

[8] Bearman, P.W., Wadcock, A.J., 1973. The interaction between a pair of circular cylinders normal to a stream. Journal of Fluid Mechanics 61, 499-511.

[9] Kim, H.J., Durbin, P.A., 1988. Investigation of the flow between a pair of circular cylinders in the flopping regime. Journal of Fluid Mechanics 196, 431-448.

[10] Sumner, D., Wong, S.S.T., Price, S.J., and Paidousis, M.P., 1999. Fluid behaviour of side-by-side circular cylinders in steady cross-flow. Journal of Fluids and Structures 13, 309-338.

[11] Stoneman S. A. T., Hourigan K., Stokes A. N. and Welsh M. C., 1988, Resonant sound caused by flow past two plates in tandem in a duct. Journal of Fluid Mechanics, 192: 455-484.

[12] Howe, M.S., 1980. The dissipation of sound at an edge. Journal of Sound and Vibration 70, 407-411.

[13] Howe, M.S., 1998. Acoustics of Fluid-Structure Interactions. Cambridge University Press, 1998.

[14] Spalart, P. R. and Allmaras, S. R., 1994, One-equation turbulence model for aerodynamic flows. Recherche Aerospatiale, n 1, p 5-21. 
[15] Isaev, S.A., Baranov, P.A., Kudryavtsev, N.A., Lysenko, D.A., and Usachev, A.E., 2005, Comparative analysis of the calculation data on an unsteady flow around a circular cylinder obtained using the VP2/3 and FLUENT packages and the SpalartAllmaras and Menter turbulence models. Journal of engineering physics and thermophysics, Vol. 78, No. 6, pp. 1199-1213.

[16] Chen, L., Tu, J.Y. and Yeoh, G.H., 2003, Numerical simulation of turbulent flows behind two side-by-side cylinders, Journal of Fluids and Structures, 18: 387 - 403.

[17] Kinsler, L. E., Frey, A. R., Coppens, A. B. and Sanders, J. V., 2000, Fundamentals of acoustics, Fourth Edition, John Wiley and Sons, Inc. 